\title{
The effect of number and minimum duration of post-response-prevention escapes on the resistance to extinction of an avoidance response
}

\author{
PHILIP J. BERSH, WAYNE G. WHITEHOUSE, and JOSHUA E. BLUSTEIN \\ Temple University, Philadelphia, Pennsylvania
}

\begin{abstract}
After one-way avoidance training, rats were exposed, during avoidance response prevention, to light (CS-only) presentations or to light-shock (CS-US) pairings. Subgroups were then given 1,5 , or 10 trials during which they could escape immediately (unrestricted) or after 5 sec (restricted) by means of the previously conditioned avoidance response from a simultaneous light-shock compound. All animals were then exposed to avoidance extinction. The number of unrestricted escapes increased responding for CS-only animals, but had no significant effect on the performance of CS-US animals. Nevertheless, resistance to extinction was considerably less for CS-only animals given 10 unrestricted escapes than for CS-US animals given one unrestricted escape. One restricted escape had no more effect than one unrestricted escape for either response-prevention group. However, 5 restricted escapes elevated responding for CS-only animals to the level of CS-US animals. Extinction responding for CS-US animals increased significantly only after 10 restricted escapes. Since CS-only animals showed no further increase, resistance to extinction once more was greater for CS-US animals. These results, together with the very brief unrestricted escape latencies of CS-only animals, support a greater role for Pavlovian extinction than for response competition in the facilitation of avoidance extinction by CS-only response prevention. The fact that 10 restricted escapes were required to elevate resistance to extinction for CS-US animals over that obtained with one unrestricted escape attests to the effectiveness of Pavlovian conditioning during avoidance response prevention in elevating CS aversiveness to a near ceiling level.
\end{abstract}

The prevention of avoidance behavior during unreinforced exposure to a preshock warning signal greatly facilitates subsequent avoidance extinction (e.g., Baum, 1970; Coulter, Riccio, \& Page, 1969; Page, 1955), with the degree of facilitation proportional to the amount of warning signal exposure (Bersh \& Keltz, 1971; Shipley, Mock, \& Levis, 1971). However, the basis for the effectiveness of avoidance-response-prevention procedures is controversial (Baum, 1970; Mineka, 1979). Although several theoretical accounts have been proposed, the effect of avoidance-response prevention in reducing the subsequent resistance to extinction of avoidance behavior is commonly ascribed to the development of competing behavior (Coulter et al., 1969; Linton, Riccio, Rohrbaugh, \& Page, 1970; Page, 1955) and/or to a reduction in the aversiveness of the warning signal resulting from its presentation without shock during response prevention, that is, Pavlovian extinction (Bersh \& Paynter, 1972; Corriveau \& Smith, 1978; Monti \& Smith, 1976). Empirical evidence favoring the competing response theories has largely been

The research reported in this paper was supported by a grant-in-aid from Temple University to the first author. We wish to express appreciation to Ann L. Maliniak and Elizabeth M. Whitehouse for their technical assistance in the preparation of the manuscript. Reprint requests should be addressed to Philip J. Bersh, Department of Psychology, Temple University, Philadelphia, PA 19122. indirect, consisting of demonstrations that responseprevention treatments may hasten avoidance extinction without promoting a parallel reduction in other indices of warning-signal aversiveness (cf. Coulter et al., 1969; Mineka \& Gino, 1979). Similarly, Marrazo, Riccio, and Riley (1974) reported equivalent facilitation of avoidance extinction for groups of rats exposed either to the warning signal alone or to warning-signal/shock pairings during response prevention. This surprising result appears to be quite damaging to the view that Pavlovian extinction during response prevention has a major role in facilitating subsequent extinction of avoidance behavior. Thus, by implication, response competition may provide the primary basis for the facilitation effect, since it apparently makes little difference whether response prevention involves unreinforced or reinforced exposure to the warning signal. Bersh, Whitehouse, and Mauro (1982) documented the importance of competing behavior in the facilitation of avoidance extinction by Pavlovian conditioning during response prevention. In their experiments, the 5-sec shocks employed by Marazzo et al. (1974) were found to elicit unconditioned rearing and jumping during response prevention. When Pavlovian conditioning, using the warning signal as a CS, was carried out during response prevention, the CS became increasingly capable of evoking such behavior, and continued to do so during avoidance extinction, thereby ostensibly hastening the ex- 
tinction of avoidance behavior. However, Bersh et al. (1982) also found that a single post-response-prevention escape by means of the response previously required for avoidance (i.e., shuttlebox crossing) weakened or eliminated the competing behavior and led to greatly enhanced extinction responding, far more than that exhibited by control animals not exposed to response prevention. They concluded that Pavlovian conditioning with a 5 -sec shock during response prevention simultaneously conditioned the competing behavior and increased the potential control by the warning signal over the avoidance response by increasing its aversiveness. Once the masking effect of the competing behavior was reduced, the increased potential control by the warning signal was realized.

By contrast, Bersh et al. (1982) found that the single post-response-prevention escape trial did not affect the rapid avoidance extinction observed for animals exposed only to the warning signal during response prevention. This result is puzzling from the viewpoint that competing behavior acquired during response prevention is largely responsible for the facilitated avoidance extinction that follows unreinforced presentations of the warning signal during response prevention (e.g., Coulter et al., 1969; Page, 1955). The argument that the competing behavior (i.e., passivity or freezing) acquired with the conventional response-prevention treatment is somehow stronger or more difficult to disrupt than rearing appears untenable. Animals exposed to the unreinforced warning signal during response prevention exhibited very short escape latencies-much shorter than animals exposed to the Pavlovian conditioning procedure. This suggests that the failure of the escape trial to increase extinction responding for such animals reflects the relatively low level of aversiveness of the warning signal, rather than the strength of competing behavior. Apparently, one brief exposure to a simultaneous compound of warning signal and shock was not adequate to restore the aversiveness of the signal to a level sufficient to enhance extinction responding.

The present experiment pursues this possibility by investigating the effects of the number and minimum duration of post-response-prevention escape trials on the subsequent resistance to extinction of the avoidance response for animals exposed either to Pavlovian extinction or to Pavlovian conditioning during response prevention. Both variables presumably influence the aversiveness of the warning signal and/or the strength of the escape/avoidance response. Thus, one of the objectives of this study was to assess the difficulty of reinstating avoidance responding for animals exposed only to the warning signal during response prevention. Resolution of this issue may have implications for clinical exposure treatments of anxiety, phobia, and obsessive-compulsive behavior. Responseprevention research with animals has served as an experimental analogue for such treatments, in particular, the flooding treatment (cf. Baum, 1970). A related objective is to determine whether even multiple escape trials are capable of increasing resistance to avoidance extinction in animals previously exposed to Pavlovian extinction during response prevention to the levels manifested by animals exposed to the Pavlovian conditioning treatment. If the problem is one of release of the avoidance response from competing behavior, multiple post-response-prevention escapes, even though they involve brief exposures to a simultaneous warning-signal/shock compound, should elevate extinction responding to the level of animals given Pavlovian conditioning during response prevention. On the other hand, if the problem is one of the low aversiveness of the warning signal, multiple exposures of longer duration may be necessary to eliminate the difference in extinction responding. Although simultaneous conditioning is less effective than forward conditioning, it has been shown to produce conditioning to a significant degree when an aversive US is employed (Burkhardt \& Ayres, 1978; Heth \& Rescorla, 1973; Mahoney \& Ayres, 1976). Furthermore, the strength of conditioning increases with duration of exposure to the simultaneous compound, at least over a limited range (Burkhardt \& Ayres, 1978).

\section{Subjects \\ One hundred and twenty male albino rats, obtained from the Holtzman Company and weighing $250-300 \mathrm{~g}$, served as subjects. The rats were housed in individual cages and were permitted free access to food and water.}

METHOD

\section{Apparatus}

All phases of the experiment were carried out in a Lehigh Valley Electronics shuttlebox $(27.3 \mathrm{~cm}$ high $\times 21.6 \mathrm{~cm}$ wide $\times 48.3 \mathrm{~cm}$ long), modified to function as a one-way avoidance apparatus. Both compartments had floors consisting of stainless steel grids, $0.24 \mathrm{~cm}$ in diameter and spaced $0.87 \mathrm{~cm}$ apart. Scrambled constant-current shock, measuring $0.80 \mathrm{~mA}$ at the grids, was supplied to the floor of only one of the compartments by a BRS-Foringer Model 2903 shocker/scrambler. The warning signal consisted of the onset of a 7.5-W houselight, mounted on the end wall of each compartment. A manually operated opaque door separated the two equal-size and identically appearing compartments. A clear Plexiglas door could also be lowered manually to prevent crossing from one compartment to the other when the opaque door was raised. During all experimental phases, the room housing the shuttlebox was unilluminated and noise-free. Data collection was accomplished by solid state equipment located in an adjacent room.

\section{Procedure}

The experimental design was a $2 \times 3 \times 2$ factorial. One factor was the avoidance response-prevention treatment. Group CS-only was exposed to unreinforced presentations of the warning signal (light) during response-prevention trials, whereas Group CS-US was exposed to Pavlovian conditioning (light-shock pairings) on such trials. A second factor was the number of post-response-prevention escape trials $(1,5$, or 10) administered prior to the avoidance extinction phase. The third factor was the minimum duration of exposure to the simultaneous lightshock compound on post-response-prevention escape trials, with the levels of this factor being either 0 sec (unrestricted escape) or $5 \mathrm{sec}$ (restricted escape).

Acquisition. Following a 5-min period of adaptation to the dark apparatus, each animal was placed in the shock compartment with the opaque door closed. Two to $10 \mathrm{sec}$ later, the light (warning signal) was presented simultaneously with the raising of the door. Five seconds following light onset, shock began and continued until the animal crossed to the safe compartment, the crossing response terminating both light and shock. The animal was immediately removed to a retaining cage 
for the 40-sec intertrial interval (ITI). Crossing within $5 \mathrm{sec}$ after light onset terminated the warning signal and avoided the shock. Training was continued until the animal met a criterion of three consecutive avoidance responses.

Response prevention. Following the last acquisition trial, animals were assigned randomly to either the CS-only or CS-US treatment condition. Eight avoidance response-prevention trials were carried out, with the Plexiglas barrier now blocking access to the safe compartment. A 40-sec ITI, during which the animal was confined to a retaining cage, separated each of the response-prevention trials, the latter being $10 \mathrm{sec}$ in duration. For rats in Group CS-only, trials began with the raising of the opaque door and the simultaneous onset of the light CS, which then continued for $10 \mathrm{sec}$. Since the animals were unable to leave the shock compartment, exposure to the CS occurred only in this compartment. Response-prevention trials were identical for rats in Group CS-US, except that shock was also delivered during the final $5 \mathrm{sec}$ of the CS on each trial.

Escape from light-shock compound. Following response prevention, rats from the CS-only and CS-US treatment conditions were randomly designated to receive 1,5 , or 10 trials, during which the earlier acquired crossing response now served to terminate a simultaneous compound of light and shock. For half of the animals in each escape condition, the Plexiglas barrier was removed prior to the beginning of the escape trial (unrestricted escape); for the remaining animals, the Plexiglas barrier was removed only after $5 \mathrm{sec}$ of exposure to the light-shock compound on each escape trial (restricted escape). Trials began with the raising of the opaque door and the simultaneous onset of light and shock, and terminated only when the animal crossed to the safe compartment. As in previous phases, the 40 -sec ITI was spent in the retaining cage.

Avoidance extinction. Forty seconds after the last escape trial, avoidance extinction began. No shocks were programmed during extinction trials. The CS was presented simultaneously with the raising of the opaque door, and continued for $10 \mathrm{sec}$. The occurrence of a crossing response within $10 \mathrm{sec}$ of CS onset terminated the CS and ended the trial. The animal was immediately removed to the retaining cage for the 40-sec ITI. If an animal failed to cross within $10 \mathrm{sec}$ after CS onset, the CS terminated automatically and the animal was removed for the ITI. Extinction was carried out until the animal failed to cross within $10 \mathrm{sec}$ of CS onset on three consecutive trials.

\section{RESULTS}

Avoidance conditioning was equivalent for all groups. A one-way analysis of variance (ANOVA) revealed no reliable intergroup differences $(F<1.0)$ in the number of trials required to meet the acquisition criterion.

\section{Extinction Performance}

Figure 1 graphs the mean number of trials to the avoidance extinction criterion (exclusive of the criterion trials themselves) for each postacquisition treatment condition. The left panel shows the data for groups given unrestricted post-response-prevention escape trials, and the right panel shows the data for groups exposed to $5 \mathrm{sec}$ of shock before escape was possible. Inspection of the left panel indicates that, with unrestricted escape trials, the mean number of extinction responses for animals exposed to CS-only during response prevention increased linearly with the number of escape trials. Although comparisons with the results of the earlier experiment (Bersh et al., 1982) are risky, it appears that 10 post-responseprevention escapes were sufficient to elevate extinction responding to a level at least equal to that for control animals (i.e., not exposed to response prevention) in the

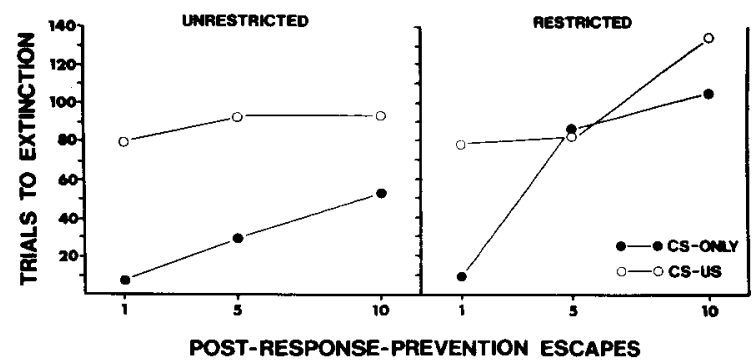

Figure 1. Mean trials to avoidance extinction for groups exposed to CS-only and CS-US response-prevention treatments as a function of number $(1,5$, or 10$)$ and minimum duration of post-responseprevention escape trials. The left panel displays the data for the unrestricted escape (i.e., 0-sec minimum duration) condition, and the right panel shows complementary data for the restricted escape (i.e., 5-sec minimum duration) condition.

earlier experiment. Little change in extinction responding was evident for CS-US animals with increases in the number of unrestricted escape trials. Nevertheless, resistance to extinction of the avoidance response for CS-US animals remained substantially greater than that for CS-only animals. In fact, even 10 unrestricted escapes for CS-only animals produced far less extinction responding than that manifested by CS-US animals given only a single unrestricted escape.

The right panel of Figure 1 indicates that one escape with a 5-sec minimum shock exposure had no more effect upon extinction responding for CS-only and CS-US animals than one unrestricted escape. On the other hand, 5 restricted escapes for CS-only animals increased their extinction responding to the same level as that for CS-US animals given 5 restricted escapes. However, because of the considerable increase in resistance to extinction manifested by the CS-US animals, the comparability of performance did not extend to 10 restricted escapes.

These observations were confirmed statistically by a three-way (response-prevention treatment $x$ number of escapes $\times$ minimum duration of escape trial) ANOVA. Accordingly, reliable main effects were obtained for treatment $[\mathrm{F}(1,108)=99.8, \mathrm{p}<.001]$, number of escapes $[\mathrm{F}(2,108)=45.1, \mathrm{p}<.001]$, and minimum duration $[F(1,108)=26.7, p<.001]$. In addition, the third-order interaction was significant $[F(2,108)=5.0, p<.01]$. Simple-effects $F$ tests revealed that CS-US animals exposed to 1,5 , or 10 unrestricted post-response-prevention escape trials emitted reliably more responses during avoidance extinction than equivalently treated CS-only animals $[F(1,108)=41.9,32.0$, and 13.5 , respectively, all ps $<.001]$. Following restricted post-responseprevention escape trials, CS-US animals again responded significantly more during avoidance extinction than CSonly animals when either $1[\mathrm{~F}(1,108)=39.2, \mathrm{p}<.001]$ or $10[\mathrm{~F}(1,108)=6.99, \mathrm{p}<.05]$ escapes were given. However, no difference was found between CS-only and CS-US animals following 5 restricted escape trials $(\mathrm{F}<1.0)$. Newman-Keuls tests $(\alpha=.05)$ revealed that 
CS-only animals showed significantly greater resistance to extinction following 10 unrestricted escape trials than after 1 or 5 unrestricted escapes. No reliable difference was found between CS-only animals given 1 or 5 unrestricted escape trials. Nor were reliable diffferences obtained for CS-US animals as a function of the number of unrestricted escape trials. CS-only animals exposed to 5 or 10 restricted escape trials were reliably more resistant to extinction than CS-only animals given only one such trial. The difference in extinction responding between CS-only animals receiving 5 and those receiving 10 restricted escape trials was not reliable. CS-US animals showed significantly greater resistance to extinction if given 10 than if given either 1 or 5 restricted escape trials; the groups exposed to the latter treatments did not differ significantly.

Comparisons of extinction performance following unrestricted vs. restricted escape indicated that although CSonly animals given restricted escape involving 5 or 10 trials were significantly more resistant to extinction than CS-only animals given unrestricted escape $[F(1,108)=$ 27.2 and 22.2 , respectively, ps $<.001$ ], no reliable difference was apparent with 1 escape trial. Similarly, the differences were not reliable for CS-US animals given 1 or 5 escape trials. At 10 escape trials, however, CS-US animals showed significantly greater resistance to avoidance extinction with the restricted condition $[\mathrm{F}(1,108)=$ 13.6, $\mathrm{p}<.001]$.

\section{Escape Performance}

Two separate analyses were applied to data for performance during the post-response-prevention escape trials. In both cases, in order to reduce heterogeneity of variance, the data are expressed in the form of escape speed rather than latency. Table 1 presents mean escape speed on the first post-response-prevention trial, based on the data for all groups collapsed over the number of escape trials. As confirmed by simple effects tests, CS-only animals in the unrestricted condition were faster to escape than CS-US animals, both when escape for the latter animals was unrestricted $[F(1,108)=17.2, p<.001]$ and when escape was restricted $[F(1,108)=23.6, p<.001]$. The escape condition, on the other hand, failed to affect differentially the escape speed of CS-US animals. Furthermore, there was no reliable difference in escape speed between CS-only and CS-US animals given restricted escape trials.
Table 1

Mean Escape Speed (in Seconds) on First Post-Response-Prevention Escape Trial

\begin{tabular}{lccccc}
$\begin{array}{c}\text { Response- } \\
\text { Prevention } \\
\text { Treatment }\end{array}$ & \multicolumn{2}{c}{ Unrestricted } & & \multicolumn{2}{c}{ Restricted } \\
\cline { 2 - 3 } \cline { 5 - 6 } & Mean & SD & & Mean & SD \\
\hline CS-Only & 1.74 & 1.25 & & 0.65 & 0.26 \\
CS-US & 0.46 & 0.33 & & 0.40 & 0.41 \\
\hline
\end{tabular}

Table 2 presents mean escape speed for the first and last escape trial and, therefore, includes data only for animals given multiple escape trials. ANOVA indicated that when escape was unrestricted, escape speed was reliably greater on the last trial than on the first trial for both the 5- and 10-trial groups $[F(1,72)=7.6$ and 35.8 , respectively, ps $<.01]$. On the other hand, when escape was restricted, no reliable change in speed occurred from the first to the last trial. Animals given 10 unrestricted escape trials were faster to escape on the last trial than animals receiving restricted trials $[F(1,72)=52.8$, $\mathrm{p}<.001$ ], but no reliable diffference was found for the 5-trial groups. With unrestricted escape, 10 trials produced reliably greater last-trial speed than did 5 trials $[F(1,72)=15.6, p<.001]$. By contrast, restricted escape resulted in reliably slower last-trial speed with 10 trials than with 5 trials $[\mathrm{F}(1,72)=4.4, \mathrm{p}<.05]$.

\section{DISCUSSION}

The results reported by Bersh et al. (1982) when animals received one post-response-prevention escape were replicated in the present experiment. That is, CSonly animals showed little resistance to avoidance extinction, whereas CS-US animals responded at a high level during extinction. Furthermore, extinction responding failed to increase for CS-only animals, even when escape was possible only after 5 sec of shock exposure. As in the earlier experiment, CS-only animals free to escape upon shock onset were reliably quicker to escape on the first trial than CS-US animals. This raises the possibility that the difference in resistance to avoidance extinction manifested by CS-only and CS-US animals was due to the difference in shock exposure on the escape trial. However, when the single escape was restricted, the same difference in extinction responding was found, despite the fact that escape speeds were not reliably different for the

Table 2

Mean Escape Speed (in Seconds) on First and Last Post-Response-Prevention Escape Trials Involving Multiple (5 and 10) Escapes

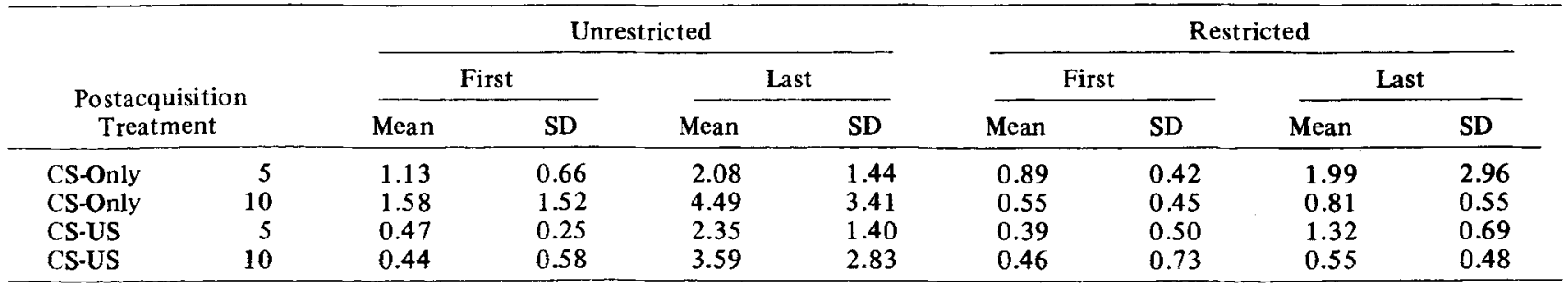


two groups. The implication is clear, therefore, that the difference in resistance to extinction was determined by the difference in response-prevention treatment.

Multiple escapes by means of the response previously required for avoidance may increase extinction responding for several reasons: (1) Repeated exposures to the simultaneous light-shock compound may increase the aversiveness of the CS and, therefore, its potential control over the avoidance response. It is also possible that exposures to the simultaneous light-shock compound add to the aversiveness of the context (often labeled "contextual fear' '). However, the light presumably overshadows the context to some degree and thereby limits contextual "fear" conditioning (e.g., Baker, Mercier, Gable, \& Baker, 1981). (2) Shock escape provides negative reinforcement for the response. When escape was unrestricted, the combination of these two factors increased extinction responding for CS-only animals to the level (i.e., 25-35 responses) consistently obtained for control rats (i.e., not exposed to response prevention) in our laboratory (e.g., Bersh \& Miller, 1975; Bersh et al., 1982), but not to the level of the CS-US animals. On the other hand, even 10 unrestricted escapes had no further reliable incremental effect on extinction responding for CS-US animals. When escape was restricted, 5 escapes greatly elevated extinction responding for CS-only animals, but did not add to such responding for CS-US animals, so that the two groups showed equal resistance to avoidance extinction. This suggested that Pavlovian conditioning during response prevention might have increased the resistance to extinction of the avoidance response to its ceiling. However, when given 10 restricted escapes, CS-US animals did show a further reliable increase in extinction responding. Since the same treatment did not show an analogous effect for CS-only animals, resistance to avoidance extinction once more was greater for the CS-US group.

It is not entirely possible to disentangle the relative contributions of CS aversiveness and negative reinforcement to the elevation of extinction responding for CS-only animals. This is particularly so because the magnitude of negative reinforcement provided by CS termination during avoidance extinction will presumably covary with CS aversiveness. Furthermore, the substantially greater influence of multiple restricted escapes than of multiple unrestricted escapes may reflect the contribution of the difference in duration of CS-shock exposure to CS aversiveness and/or the increased strengthening of the crossing response itself by the greater negative reinforcement provided by escape from a longer duration shock. Several studies have demonstrated that shock aversiveness is a joint function of intensity and duration (Church, Raymond, \& Beauchamp, 1967; Leander, 1973). However, it is reasonable to suggest that the primary factor in determining resistance to extinction was the aversiveness of the CS. As noted, one restricted escape had no more effect than one unrestricted escape on extinction responding by CS-only or CS-US animals. Moreover, when escape was restricted, the very much greater resistance to avoidance extinction manifested by the CS-US group could not be attributed to a difference in shock exposure or negative reinforcement on the escape trial, because escape speed was no less than that exhibited by the CS-only group. Accordingly, it must have been due to the difference in the response-prevention treatment. By its very nature, response prevention precludes negative reinforcement for the avoidance response. Indeed, it leads to behavior antagonistic to the avoidance response for CSUS animals, behavior that impedes extinction responding in the absence of at least one post-response-prevention escape (Bersh et al., 1982). Therefore, the difference in extinction responding when post-response-prevention escapes were provided was presumably due to the increased aversiveness of the CS resulting from Pavlovian conditioning for Group CS-US and to the decreased aversiveness of the CS resulting from Pavlovian extinction during response prevention for Group CS-only (cf. Bersh \& Paynter, 1972; Monti \& Smith, 1976). The fact that 10 unrestricted or 5 restricted escapes failed to increase extinction responding for CS-US animals indicates that the combination of further light-shock exposures and/or negative reinforcements was inadequate to enhance the effect of the already high level of CS aversiveness produced by Pavlovian conditioning during response prevention. Apparently, 10 restricted escapes with their concomitant longer exposures to the CS-shock compound were required before CS aversiveness could exceed the level established by Pavlovian conditioning during the preceding response-prevention phase. In this connection, it is noteworthy that escape remained relatively slow throughout the course of the 10 restricted trials, whereas after the initial trial escape became quite rapid when not restricted. In fact, the unrestricted escape speed for CSUS animals equaled that for CS-only animals within a trial or two, so that later escapes involved very brief exposures to the CS-shock compound, too brief to add materially to the already high level of CS aversiveness for CS-US animals. On the other hand, given the low level of CS aversiveness for CS-only animals, repeated brief exposures over the course of 10 unrestricted escape trials might have reliably increased CS aversiveness, although hardly to the level of CS-US animals.

The possibility should also be considered that the total duration of shock exposure was a determinant of differences in extinction responding. It is obvious that there is no way to vary the number and minimum duration of shock exposures during post-response-prevention escapes without covariation of total duration of shock exposure. Furthermore, for any escape treatment, total shock exposure for CS-US animals must exceed that for CS-only animals by at least $40 \mathrm{sec}$, the duration of responseprevention shock exposure for the former group. Nevertheless, earlier findings (Bersh et al., 1982) clearly demonstrated that the type of response-prevention treatment and the presence or absence of a post-responseprevention escape, rather than the total duration of shock 
exposure, were decisive for subsequent extinction performance. Indeed, groups of rats exposed to identical shocks during response prevention differed significantly in resistance of the avoidance response to extinction, depending on whether those shocks were signaled or not. Thus, given a single unrestricted post-response-prevention escape, light-shock animals responded far more often during extinction than did shock-only animals; without such an escape, they responded far less often. In fact, regardless of whether an escape trial was given, animals exposed to unsignaled shocks during response prevention subsequently exhibited levels of responding during avoidance extinction that were comparable to those of control animals not given response prevention. It might also be expected that increased shock exposure under conditions in which escape and/or avoidance were prevented (i.e., during the first $5 \mathrm{sec}$ of restricted escape and for CS-US animals during response prevention) would produce stronger freezing or other competing behavior. Bersh et al. (1982) found that competing behavior in the form of rearing and jumping did develop for CS-US animals during response prevention. Furthermore, the finding that escape speeds decreased in the present experiment for both CS-only and CS-US animals when escape was restricted is also consistent with the possible development of competing behavior. Thus, the argument might be made that multiple restricted escapes, in view of their concomitantly greater durations of shock, may promote stronger freezing or rearing tendencies than would the same number of unrestricted escapes. Stronger competing behavior obviously would be expected to reduce responding during avoidance extinction. Nevertheless, the present results indicate clearly that multiple restricted post-response-prevention escapes enhanced avoidance extinction responding for CS-only animals and, when sufficiently numerous, also increased the already high level of extinction responding for CS-US animals. If competing responses had developed more strongly for animals exposed to restricted escapes, they certainly failed to influence resistance to extinction of the avoidance response in the usual manner. Based upon these considerations, it seems reasonable to discount differences in total shock exposure per se as the basis for differences in subsequent extinction responding.

At any rate, the results of the present experiment fail to support response competition as the sole, or even primary, basis for the facilitation of avoidance extinction by a CS-only response-prevention treatment. Nor was the increase in extinction responding for CS-only animals as a function of number and minimum duration of escape trials attributable to the elimination of competing behavior. In both this and the previous experiment (Bersh et al., 1982), one post-response-prevention escape was sufficient to eliminate competing behavior for CS-US animals. Competing behavior, if it existed at all for CS-only animals, was considerably weaker, as indicated by escape speed for the first escape trial. Accordingly, no more than one escape should have been necessary to eliminate it.
Yet, even five unrestricted escapes failed to increase extinction responding reliably. By implication, then, Pavlovian extinction is a more significant factor in the facilitation of avoidance extinction by a CS-only responseprevention treatment than a response competition theory suggests, and is primarily responsible for the fact that multiple post-response-prevention escapes are needed to enhance the resistance to extinction of the avoidance response.

\section{REFERENCES}

Baker, A. G., Mercieh, P., Gabel, J., \& Baker, P. A. (1981). Contextual conditioning and the US preexposure effect in conditioned fear. Journal of Experimental Psychology: Animal Behavior Processes, 7, 109-128.

BAUM, M. (1970). Extinction of avoidance responding through response prevention (flooding). Psychological Bulletin, 74, 276-284.

Bersh, P. J., \& Keltz, J. R. (1971). Pavlovian reconditioning and the recovery of avoidance behavior in rats after extinction with response prevention. Journal of Comparative and Physiological Psychology, 76, 262-266.

Bersh, P. J., \& MilleR, K. (1975). The influence of shock during response prevention upon resistance to extinction of an avoidance response. Animal Learning \& Behavior, 3, 140-142.

Bersh, P. J., \& Paynter, W. E. (1972). Pavlovian extinction in rats during avoidance response prevention. Journal of Comparative and Physiological Psychology, 78, 255-259.

Bersh, P. J., Whitehouse, W. G., \& Mauro, B. C. (1982). Pavlovian processes and response competition as determinants of avoidance response-prevention effects. Learning and Motivation, 13, 113-134.

Burkhardt, P. E., \& Ayres, J. J. B. (1978). CS and US duration effects in one-trial simultaneous fear conditioning as assessed by conditioned suppression of licking in rats. Animal Learning \& Behavior, 6, 225-230.

Church, R. M., Raymond, G. A., \& Beauchamp, R. D. (1967). Response suppression as a function of intensity and duration of a punishment. Journal of Comparative and Physiological Psychology, 63, 39-44.

Corriveau, D. P., \& Smith, N. F. (1978). Fear reduction and "safety-test" behavior following response-prevention: A multivariate analysis. Journal of Experimental Psychology: General, 107, 145-158.

Coulter, X., Riccio, D. C., \& Page, H. A. (1969). Effects of blocking an instrumental avoidance response: Facilitated extinction but persistence of "fear." Journal of Comparative and Physiological Psychology, 68, 377-381.

Heth, C. D., \& Rescorla, R. A. (1973). Simultaneous and backward fear conditioning in the rat. Journal of Comparative and Physiological Psychology, 82, 434-443.

LEANDE R, J. D. (1973). Shock intensity and duration interactions on free-operant avoidance behavior. Journal of the Experimental Analysis of Behavior, 19, 481-490.

Linton, J., Riccio, D. C., Rohranugh, M., \& Page, H. A. (1970). The effects of blocking an instrumental avoidance response: Fear reduction or enhancement? Behavior Research and Therapy, 8, 267-272.

Mahoney, W. J., \& AYres, J. J. B. (1976). One-trial simultaneous and backward fear conditioning as reflected in conditioned suppression of licking in rats. Animal Learning \& Behavior, 4, 357-362.

Marazzo, M. J., Riccio, D. C., \& Riley, J. (1974). Effects of Pavlovian conditioned stimulus-unconditioned stimulus pairing during avoidance response-prevention trials in rats. Journal of Comparative and Physiological Psychology, 86, 96-100. 
MineKA, S. (1979). The role of fear in theories of avoidance learning, flooding, and extinction. Psychological Bulletin, 86, 985-1010.

Mine Ka, S., \& Gino, A. (1979). Dissociative effects of different types and amounts of nonreinforced CS exposure on avoidance extinction and the CER. Learning and Motivation, 10, 141-160.

Monti, P. M., \& Smith, N. F. (1976). Residual fear of the conditioned stimulus as a function of response prevention after avoidance or classical defensive conditioning in the rat. Journal of Experimental Psychology: General, 105, 148-162.

Page, H. A. (1955). The facilitation of experimental extinction by response-prevention as a function of the acquisition of a new response. Journal of Comparative and Physiological Psychol$o g y, 48,14-16$.

Shipley, R. H., Mock, L. A., \& Levis, D. J. (1971). Effects of several response prevention procedures on activity, avoidance responding, and conditioned fear in rats. Journal of Comparative and Physiological Psychology, 77, 256-270.

(Manuscript received January 31, 1984; revision accepted for publication May 22, 1984.) 